\title{
Korteweg-de Vries description of Helmholtz-Kerr dark solitons
}

\author{
J M Christian ${ }^{1}$, G S McDonald ${ }^{1}$ and P Chamorro-Posada ${ }^{2}$ \\ ${ }^{1}$ Joule Physics Laboratory, School of Computing, Science and Engineering, \\ Institute for Materials Research, University of Salford, Salford M5 4WT, UK \\ 2 Departmento de Teoría de la Señal y Comunicaciones e Ingeniería Telemática, Universidad de \\ Valladolid, ETSI Telecomunicación, Campus Miguel Delibes s/n, 47011 Valladolid, Spain
}

Received 16 August 2006, in final form 6 October 2006 Published 30 November 2006

Online at stacks.iop.org/JPhysA/39/15355

\begin{abstract}
A wide variety of different physical systems can be described by a relatively small set of universal equations. For example, small-amplitude nonlinear Schrödinger dark solitons can be described by a Korteweg-de Vries (KdV) equation. Reductive perturbation theory, based on linear boosts and Gallilean transformations, is often employed to establish connections to and between such universal equations. Here, a novel analytical approach reveals that the evolution of small-amplitude Helmholtz-Kerr dark solitons is also governed by a KdV equation. This broadens the class of nonlinear systems that are known to possess KdV soliton solutions, and provides a framework for perturbative analyses when propagation angles are not negligibly small. The derivation of this KdV equation involves an element that appears new to weakly nonlinear analyses, since transformations are required to preserve the rotational symmetry inherent to Helmholtz-type equations.
\end{abstract}

PACS numbers: 42.65.- $\mathrm{k}, 42.65 . \mathrm{Tg}, 05.45 . \mathrm{Yv}$

\section{Introduction}

Solitons are self-confined waves that propagate stably in nonlinear media. Such localized structures appear in planar optical waveguides when the (linear) spreading due to diffraction is balanced exactly by (nonlinear) narrowing from medium self-focusing. The evolution of Kerr spatial solitons has been routinely described by the nonlinear Schrödinger (NLS) equation $[1,2]$. This paraxial model describes axial and near-axial wave propagation, arising from the interplay of one-dimensional diffraction and host refractive index changes that vary linearly with the local beam intensity. For a focusing Kerr medium, bright spatial solitons exist subject to vanishing-asymptotic boundary conditions [3]. These solutions are highly localized in the transverse dimension and have a sech-shaped profile. For defocusing media, the NLS equation supports dark solitons that involve a tanh-shaped component [4]. This latter class of 
solution has a transverse intensity distribution consisting of a uniform (non-zero) background modulated by a localized 'dip'. When this dip does not result in a point of zero intensity, the dark soliton is termed 'grey'.

It is known that small-amplitude NLS dark solitons are governed by the Korteweg-de Vries $(\mathrm{KdV})$ equation [5]. The dynamics of such solitons in the presence of various perturbative effects can therefore be analysed in terms of KdV theory [6]. This general property has, for example, been exploited to analyse temporal solitons in optical fibres, where stimulated Raman scattering may perturb the nonlinear refractive index change [7-9]. Other analyses of small-amplitude dark pulses in optical fibres have shown that KdV-type equations govern dynamics near the zero-dispersion point $[10,11]$. The multiple-scales formalism employed in these analyses has also been applied to a wide variety of other nonlinear systems [12]. For example, waves in electrical transmission lines and those on 1D atomic chains can be described by $\mathrm{KdV}$ equations. The connection between solitons of strongly dispersive and weakly nonlinear (e.g. NLS, where the linear-dispersive term involves a second-order derivative) and weakly dispersive and weakly nonlinear (e.g. $\mathrm{KdV}$, where the linear-dispersive term involves a third-order derivative) systems highlights a certain universality that exists for nonlinear wave equations. In particular, it has been assumed that the integrability of both the $\mathrm{KdV}$ and NLS equations is the principle reason for such a mathematical connection.

Weakly nonlinear analysis does not only furnish new analytical frameworks, but it can also provide new insights and establish trans-disciplinary activity. For example, temporal vector solitons, in the so-called standard configuration of an optical fibre [13], have underlying universal equations in the form of the well-known Zakharov equations of plasma physics. Ion-acoustic waves in plasmas are themselves described by universal nonlinear equations $[14,15]$. Coullet et al [16] showed that the laser Maxwell-Bloch equations may be reduced to a complex Ginzburg-Landau equation. This particular contribution underpinned advances concerning optical vortices and optical pattern formation [16, 17], and in general laser physics [18].

Here, we consider spatial solitons in two-dimensional planar waveguides, where there is a longitudinal dimension and a single (effective) transverse dimension. Such solitons are expected to play a central role as stable information units in future optical applications involving, for example, photonic switching, optical interconnects, beam steering and information/image processing. The dominant theme of this paper concerns the obliquepropagation (i.e. off-axis) aspects of spatial solitons. In particular, we demonstrate that Helmholtz-Kerr dark solitons (strictly speaking 'solitary waves'), which are exact analytical solutions of a non-integrable wave equation, are also described by a $\mathrm{KdV}$ equation in their small-amplitude limit. The multiple-scales analysis employed here involves a novel transformation that is necessary in order to respect the spatial symmetry inherent to Helmholtztype wave equations. This transformation corresponds physically to a rotation of the unscaled coordinate axes, rather than a Gallilean linear boost. Our framework is thus fundamentally different from those considered by other authors [5-18], whose analyses are based upon Gallilean-type invariance laws.

\section{Helmholtz dark solitons}

In optics, one frequently encounters the paraxial approximation. A spatial beam is paraxial if all three of the following criteria are satisfied: it must be (i) broad in comparison to the optical wavelength, (ii) of sufficiently low intensity and (iii) propagating either along, or at negligible angles to, the reference axis. If all three conditions are not met simultaneously, then the paraxial approximation fails and the beam may be described as 'non-paraxial'. 
By relaxing conditions (i) and (ii), one defines the ultra-narrow beam (or 'sub-wavelength') type of non-paraxial regime. In this case, one must take full account of the vectorial nature of the electric field, and allow for additional contributions from rapid spatial variations in the nonlinear polarization [19-21]. In this paper, we are concerned solely with the Helmholtz non-paraxial scenario. This involves broad, moderately intense optical beams propagating and interacting at arbitrarily large angles with respect to the longitudinal (reference) direction [22]. In other words, criteria (i) and (ii) are always satisfied rigorously, but (iii) is relaxed. For uniform media, and with the explicit assumption of broad beams, one may consider the refractive-index distributions within the scalar approximation. In this regime, higher order perturbative correction terms in the governing wave equation are unnecessary. Rather, the Helmholtz angular correction to paraxial theory, which is purely geometrical in nature, can be of any order even though the optical beam is always broad [22-24]. When oblique evolution is the sole source of non-paraxiality, the electric field can be regarded as predominantly transverse (polarized, typically, in the $x-z$ plane of the waveguide), and a nonlinear Helmholtz (NLH) equation captures the full description of wave propagation. By omitting the slowly varying envelope approximation, the transverse $(x)$ and longitudinal $(z)$ coordinates occur symmetrically and, as a consequence, light is allowed to diffract in both these dimensions. Since there is no physical distinction between $x$ and $z$ in uniform media, the wave equation retains the inherent spatial symmetry [22].

For a Kerr medium, where the refractive index distribution $n=n_{0}+n_{2}|E|^{2}$ is well approximated by $n^{2} \approx n_{0}^{2}+2 n_{0} n_{2}|E|^{2}$, the NLH equation may be written in a form more compatible with the familiar cubic NLS model,

$$
\kappa \frac{\partial^{2} u}{\partial \zeta^{2}}+\mathrm{i} \frac{\partial u}{\partial \zeta}+\frac{1}{2} \frac{\partial^{2} u}{\partial \xi^{2}} \pm|u|^{2} u=0 .
$$

Here, the spatial coordinates are scaled as $\zeta=z / L_{D}$ and $\xi=\sqrt{2} x / w_{0}$, where $L_{D}=k w_{0}^{2} / 2$ is the diffraction length of a Gaussian beam with waist $w_{0} . \kappa=1 / k^{2} w_{0}^{2}=\left(\lambda / w_{0}\right)^{2} / 4 \pi^{2} n_{0}^{2}$ is the non-paraxial parameter and $u$ is the normalized electric field envelope $E(x, z)=$ $E_{0} u(x, z) \exp (\mathrm{i} k z)$, where $E_{0}=\left(n_{0} / k\left|n_{2}\right| L_{D}\right)^{1 / 2}, k=2 \pi n_{0} / \lambda, \lambda$ is the optical wavelength, $n_{0}$ is the linear refractive index and $n_{2}$ is the Kerr coefficient. The \pm sign corresponds to a focusing/defocusing medium, respectively.

The Helmholtz operator $\kappa \partial_{\zeta \zeta}$ leads to several physical features absent from paraxial theory. These have been discussed in earlier publications, where exact analytical bright [22, 23] and dark [24] soliton solutions were first reported. Exact analytical soliton solutions are now known for a wide range of scalar and vector Helmholtz-type wave equations [25]. These equations and their solutions could provide a key analytical platform for modelling novel off-axis contexts (oblique propagation and multiplexing) involving beams in various types of nonlinear media. The new solution families capture non-trivial corrections to the paraxial beam profile (angular beam broadening) and to the beam phase. Another attractive feature of NLH-based models is that they can support both travelling- and standing-wave solutions. They are therefore capable of describing accurately the propagation and interaction of multiple beams at arbitrary angles with respect to the reference direction [26].

An exact Helmholtz dark soliton is [24]

$$
\begin{aligned}
& u(\xi, \zeta)=u_{0}[A \tanh \Theta+\mathrm{i} F] \exp \left[\mathrm{i} \sqrt{\frac{1-4 \kappa u_{0}^{2}}{1+2 \kappa V^{2}}}\left(-V \xi+\frac{\zeta}{2 \kappa}\right)\right] \exp \left(-\mathrm{i} \frac{\zeta}{2 \kappa}\right), \\
& \Theta(\xi, \zeta)=\frac{u_{0} A(\xi+W \zeta)}{\sqrt{1+2 \kappa W^{2}}}
\end{aligned}
$$




$$
\begin{aligned}
& W=\frac{V-V_{0}}{1+2 \kappa V V_{0}}, \\
& V_{0}=\frac{u_{0} F}{\sqrt{1-\left(2+F^{2}\right) 2 \kappa u_{0}^{2}}},
\end{aligned}
$$

where $A^{2}+F^{2}=1$. The structure of this soliton has similarities with its paraxial counterpart. $V$ is the transverse velocity of the nonlinear plane-wave background field, defined by the choice of reference direction, and $V_{0}$ is the intrinsic velocity associated with greyness of the localized soliton component. $W$ is the net velocity, and is related to velocity summation in the unscaled reference frame. The velocities $\left(V, V_{0}\right.$ and $\left.W\right)$ in the scaled $(\xi, \zeta)$ reference frame and propagation angles $\left(\theta, \theta_{0}\right.$ and $\theta-\theta_{0}$, respectively) in the laboratory $(x, z)$ frame are related through $\tan \theta=\sqrt{2 \kappa} V, \tan \theta_{0}=\sqrt{2 \kappa} V_{0}$ and $\tan \left(\theta-\theta_{0}\right)=\sqrt{2 \kappa} W$. The sizes of the contributions $\kappa V^{2}, \kappa V_{0}^{2}$ and $\kappa W^{2}$, arising from the Helmholtz operator $\kappa \partial_{\zeta \zeta}$, are evidently determined solely by the sizes of the propagation angles $\theta$ and $\theta_{0}$. They may each, for example, be of order unity, even though for the Helmholtz type of non-paraxiality, one always has that $\kappa \ll O$ (1) (i.e. broad beams). On the other hand, the classic paraxial dark soliton solution

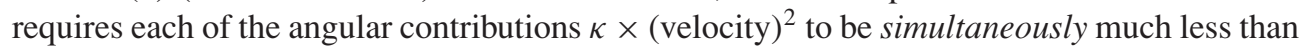
unity.

The complex beam envelope of $(2 a)$ can be recast into the form

$$
u(\xi, \zeta)=u_{0}\left[1-A^{2} \operatorname{sech}^{2} \Theta(\xi, \zeta)\right]^{1 / 2} \exp [\mathrm{i} \psi(\xi, \zeta)]
$$

where $\psi(\xi, \zeta)$ is a phase distribution. In the small-amplitude limit, where $A \ll 1$,

$$
u(\xi, \zeta) \simeq u_{0}\left[1-\frac{A^{2}}{2} \operatorname{sech}^{2} \Theta(\xi, \zeta)\right] \exp [\mathrm{i} \psi(\xi, \zeta)]
$$

It is this limit that is of particular interest for the proceeding analysis where perturbations to the nonlinear plane wave background field are quantified.

\section{Helmholtz master equations}

The defocusing NLH equation (1) has a nonlinear plane wave solution that can be found by ignoring the transverse diffraction term. Substitution of the ansatz $u(\zeta)=u_{0} \exp \left(\mathrm{i} k_{\zeta} \zeta\right)$ into the resulting wave equation yields a familiar Helmholtz auxiliary equation

$$
\kappa k_{\zeta}^{2}+k_{\zeta}+u_{0}^{2}=0 \text {. }
$$

This quadratic equation has two solutions, corresponding to on-axis $(V=0)$ forward- and backward-propagating waves. The forward solution is

$$
u(\zeta)=u_{0} \exp \left[\frac{\mathrm{i}}{2 \kappa}\left(-1+\sqrt{1-4 \kappa u_{0}^{2}}\right) \zeta\right]
$$

In the double limit $\kappa \rightarrow 0$ (broad beams) and $\kappa u_{0}^{2} \rightarrow 0$ (moderate intensities), plane wave (5) tends to that of the (paraxial) NLS equation, that is, $u(\zeta)=u_{0} \exp \left(-\mathrm{i} u_{0}^{2} \zeta\right)$. To proceed, solutions to (1) are sought in the form

$$
u(\xi, \zeta)=\left[u_{0}+a(\xi, \zeta)\right] \exp \left[\frac{\mathrm{i}}{2 \kappa}\left(-1+\sqrt{1-4 \kappa u_{0}^{2}}\right) \zeta+\mathrm{i} \phi(\xi, \zeta)\right],
$$

where the functions $a(\xi, \zeta)$ and $\phi(\xi, \zeta)$ are real perturbations to the amplitude and phase, respectively, of the nonlinear plane wave. By substituting (6) into (1), and retaining terms up to second order in the perturbations, a pair of master equations is found 
$\sqrt{1-4 \kappa u_{0}^{2}} \frac{\partial a}{\partial \zeta}+\kappa\left(u_{0}+a\right) \frac{\partial^{2} \phi}{\partial \zeta^{2}}+\frac{u_{0}+a}{2} \frac{\partial^{2} \phi}{\partial \xi^{2}}+2 \kappa \frac{\partial a}{\partial \zeta} \frac{\partial \phi}{\partial \zeta}+\frac{\partial a}{\partial \xi} \frac{\partial \phi}{\partial \xi}=0$,

$\kappa \frac{\partial^{2} a}{\partial \zeta^{2}}-\sqrt{1-4 \kappa u_{0}^{2}}\left(u_{0}+a\right) \frac{\partial \phi}{\partial \zeta}-\kappa u_{0}\left(\frac{\partial \phi}{\partial \zeta}\right)^{2}+\frac{1}{2} \frac{\partial^{2} a}{\partial \xi^{2}}-\frac{u_{0}}{2}\left(\frac{\partial \phi}{\partial \xi}\right)^{2}-2 u_{0}^{2} a-3 u_{0} a^{2}=0$.

\section{Leading-order behaviour}

To examine the leading-order behaviour of the perturbation, one retains only the linear terms in (7) so that

$$
\begin{aligned}
& \sqrt{1-4 \kappa u_{0}^{2}} \frac{\partial a}{\partial \zeta}+\kappa u_{0} \frac{\partial^{2} \phi}{\partial \zeta^{2}}+\frac{u_{0}}{2} \frac{\partial^{2} \phi}{\partial \xi^{2}}=0, \\
& \kappa \frac{\partial^{2} a}{\partial \zeta^{2}}-u_{0} \sqrt{1-4 \kappa u_{0}^{2}} \frac{\partial \phi}{\partial \zeta}+\frac{1}{2} \frac{\partial^{2} a}{\partial \xi^{2}}-2 u_{0}^{2} a=0 .
\end{aligned}
$$

From this pair of coupled equations it is straightforward to show that, at leading order, the perturbation is a phase wave in $(\xi, \zeta)$ space that satisfies the equation $\left(\partial_{\zeta \zeta}-C^{2} \partial_{\xi \xi}\right) \phi=0$. $C$ is the wave speed of these quasi-linear diffracting waves, and satisfies

$$
C^{2}=\frac{u_{0}^{2}}{1-6 \kappa u_{0}^{2}}
$$

In the limit $\kappa u_{0}^{2} \rightarrow 0$, the linear wave speed obeys $C^{2} \rightarrow u_{0}^{2}$, agreeing with the result arising from the analysis of the corresponding NLS equation [5]. Equation (9) also demonstrates that the leading-order dynamical behaviour is consistent, both physically and mathematically, with the full Helmholtz soliton solution (2), since

$$
\lim _{F \rightarrow 1} V_{0}^{2} \rightarrow C^{2}
$$

where the limit $F \rightarrow 1$ is equivalent to $A \rightarrow 0$ (the small-amplitude limit).

\section{Weakly nonlinear analysis}

To develop the description of perturbations into the weakly nonlinear regime, we undertake a multiple-scales analysis. Such a procedure entails series expansions, involving both independent and dependent variables of the system, in terms of a formal small parameter $0<\varepsilon \ll 1$. For two independent variables, such as $x$ and $z$, one typically defines

$$
\tau=\varepsilon^{p}(x-C z), \quad Z=\varepsilon^{q} z .
$$

Here, $\tau$ and $z$ are referred to as the slow variables, since large changes in $x$ and $z$ produce only small changes in $\tau$ and $z$. A suitable set of indices $(p, q)$ can be identified by analysing the linear dispersive properties of the system. The transformation above represents a linear boost (Gallilean transformation) to a frame moving at a speed $C$ along the $x$-axis. In all of the analyses cited in section 1 , the connection between slow and independent variables is based upon a Gallilean transformation [27].

The weakly nonlinear analysis of the NLH equation (1) differs fundamentally from those of paraxial NLS equations. Introducing a Gallilean transformation into the NLH framework breaks the rotational symmetry of the problem and lacks a physical correspondence with the phenomena concerned. A key objective of Helmholtz soliton theory is to eliminate such 
unphysical features and to study the implications of full spatial symmetry for more general soliton dynamics.

It can be shown that the invariance relations for the exact Helmholtz dark soliton solution (2) are given by [28]:

$$
\begin{aligned}
& \xi=\frac{\xi^{\prime}+V \zeta^{\prime}}{\sqrt{1+2 \kappa V^{2}}}, \\
& \zeta=\frac{-2 \kappa V \xi^{\prime}+\zeta^{\prime}}{\sqrt{1+2 \kappa V^{2}}} \\
& u(\xi, \zeta)=\exp \left[\frac{\mathrm{i} V \xi^{\prime}}{\sqrt{1+2 \kappa V^{2}}}+\frac{\mathrm{i}}{2 \kappa}\left(1-\frac{1}{\sqrt{1+2 \kappa V^{2}}}\right) \zeta^{\prime}\right] u^{\prime}\left(\xi^{\prime}, \zeta^{\prime}\right) .
\end{aligned}
$$

$V$ is a soliton velocity parameterizing the transformation in the scaled $(\xi, \zeta)$ frame. Equations $(10 a)$ and $(10 b)$ correspond to a rotation of the unscaled $(x, z)$ coordinate axes through an angle $\theta=\tan ^{-1}(\sqrt{2 \kappa} V)$ [22-24]. In the limit $\kappa V^{2} \rightarrow 0$, that is the angle $\theta$ becomes vanishingly small, one recovers the Gallilean invariance laws of the NLS equation exploited by other authors [5-18]. Equation (10c) expresses how the wave-field $u$ must transform in order to ensure form invariance of equation (1).

To respect rotational symmetry, the slow variables for the Helmholtz analysis are taken as

$$
\begin{aligned}
\tau & =\varepsilon \frac{\xi+C \zeta}{\sqrt{1+2 \kappa C^{2}}}, \\
Z & =\varepsilon^{3} \frac{-2 \kappa C \xi+\zeta}{\sqrt{1+2 \kappa C^{2}}} .
\end{aligned}
$$

It should be noted that $C$ is a leading-order representation of the intrinsic velocity of the Helmholtz dark soliton, since the direction of the plane wave background field is considered to be axial. Equations (11) are also consistent with the Gallilean transformation laws of the NLS equation in the limit $\kappa C^{2} \rightarrow 0$. The perturbations $a(\xi, \zeta)$ and $\phi(\xi, \zeta)$ are now expanded as asymptotic series in terms of $\varepsilon$,

$$
\begin{aligned}
& a(\xi, \zeta)=\sum_{j=0}^{\infty} \varepsilon^{2(j+1)} a_{j}(\xi, \zeta) \simeq \varepsilon^{2} a_{0}(\xi, \zeta)+\varepsilon^{4} a_{1}(\xi, \zeta)+\cdots \\
& \phi(\xi, \zeta)=\sum_{j=0}^{\infty} \varepsilon^{2 j+1} \phi_{j}(\xi, \zeta) \simeq \varepsilon \phi_{0}(\xi, \zeta)+\varepsilon^{3} \phi_{1}(\xi, \zeta)+\cdots
\end{aligned}
$$

Substituting the slow variables and the field expansions into master equations (7) and collecting together terms at $O\left(\varepsilon^{2}\right), O\left(\varepsilon^{3}\right), O\left(\varepsilon^{4}\right)$ and $O\left(\varepsilon^{5}\right)$ yields,

$C \gamma \frac{\partial a_{0}}{\partial \tau}+\frac{u_{0}}{2} \frac{\partial^{2} \phi_{0}}{\partial \tau^{2}}=0$

$C \gamma \frac{\partial a_{1}}{\partial \tau}+\frac{u_{0}}{2} \frac{\partial^{2} \phi_{1}}{\partial \tau^{2}}=-\gamma \frac{\partial a_{0}}{\partial Z}-\frac{a_{0}}{2} \frac{\partial^{2} \phi_{0}}{\partial \tau^{2}}-\frac{\partial a_{0}}{\partial \tau} \frac{\partial \phi_{0}}{\partial \tau}-\frac{2 \kappa C u_{0}}{1+2 \kappa C^{2}} \frac{\partial^{2} \phi_{0}}{\partial Z \partial \tau}$,

$C \gamma \frac{\partial \phi_{0}}{\partial \tau}+2 u_{0} a_{0}=0$

$u_{0}\left(C \gamma \frac{\partial \phi_{1}}{\partial \tau}+2 u_{0} a_{1}\right)=\frac{1}{2} \frac{\partial^{2} a_{0}}{\partial \tau^{2}}-u_{0} \gamma \frac{\partial \phi_{0}}{\partial Z}-\frac{u_{0}}{2}\left(\frac{\partial \phi_{0}}{\partial \tau}\right)^{2}-C \gamma a_{0} \frac{\partial \phi_{0}}{\partial \tau}-3 u_{0} a_{0}^{2}$, 
where $\gamma \equiv\left(1-4 \kappa u_{0}^{2}\right)^{1 / 2}\left(1+2 \kappa C^{2}\right)^{-1 / 2}$. Self-consistency requires $(13 a)$ and $(13 c)$ to be equivalent. It is straightforward to show that they are indeed equivalent if $C$ is given by (9). This encouraging result links the phase $\phi_{0}$ to the amplitude $a_{0}$ through

$$
\frac{\partial \phi_{0}}{\partial \tau}=-\frac{2 u_{0}}{C \gamma} a_{0} .
$$

Next, taking the $\tau$ derivative of (13d), and using (14), brings out the underlying KdV structure of the Helmholtz problem

$$
\begin{array}{r}
\frac{2 u_{0}^{2}}{C} \frac{\partial a_{0}}{\partial Z}+\frac{1}{2} \frac{\partial^{3} a_{0}}{\partial \tau^{3}}-6 u_{0} a_{0} \frac{\partial a_{0}}{\partial \tau}=u_{0}\left(2 u_{0} \frac{\partial a_{1}}{\partial \tau}+C \gamma \frac{\partial^{2} \phi_{1}}{\partial \tau^{2}}\right) \\
+u_{0} \frac{\partial \phi_{0}}{\partial \tau} \frac{\partial^{2} \phi_{0}}{\partial \tau^{2}}+C \gamma\left(a_{0} \frac{\partial^{2} \phi_{0}}{\partial \tau^{2}}+\frac{\partial a_{0}}{\partial \tau} \frac{\partial \phi_{0}}{\partial \tau}\right) .
\end{array}
$$

By manipulating (13b), and using (14), the first two terms on the right-hand side of (15) can be replaced by

$$
\frac{2 u_{0}^{2}}{C}\left(\frac{1-8 \kappa u_{0}^{2}}{1-4 \kappa u_{0}^{2}}\right) \frac{\partial a_{0}}{\partial Z}+6 u_{0} a_{0} \frac{\partial a_{0}}{\partial \tau} .
$$

With further algebraic manipulation, the following $\mathrm{KdV}$ equation is obtained:

$$
\frac{1}{1+2 \kappa C^{2}}\left(\frac{2 u_{0}^{2}}{C}\right) \frac{\partial a_{0}}{\partial Z}+\frac{1}{4} \frac{\partial^{3} a_{0}}{\partial \tau^{3}}-6 u_{0} a_{0} \frac{\partial a_{0}}{\partial \tau}=0 .
$$

In the limit $\kappa C^{2} \rightarrow 0$, the corresponding NLS result [5] is obtained, as should be the case. Equation (16) has the soliton solution

$$
\begin{aligned}
& a_{0}(Z, \tau)=-\left(\frac{\alpha^{2}}{2 u_{0}}\right) \operatorname{sech}^{2}[\alpha(\tau-\bar{V} Z)], \\
& \bar{V}(\kappa, \alpha)=\alpha^{2}\left(\frac{C}{2 u_{0}^{2}}\right)\left(1+2 \kappa C^{2}\right),
\end{aligned}
$$

where $\alpha$ is a free parameter and $\bar{V}$ is an effective velocity in terms of the new Helmholtz slow variables. The expression for $\bar{V}$ includes a, potentially significant, correction of $O\left(\kappa C^{2}\right)$ to its paraxial counterpart. Comparison of the KdV solution with the small-amplitude approximation (3) yields a simple relationship between the dark soliton amplitude and the formal expansion parameter $\varepsilon$. This is the same as in the paraxial case, and is given by

$$
A u_{0}=\varepsilon \alpha .
$$

Power series expansions, and use of (2), (9), (11) and (18), verify convergence of the approximate Helmholtz soliton (3) and the $\mathrm{KdV}$ solution (17) in the limit of low soliton amplitude. Moreover, the intrinsic velocity $V_{0}$, as given by $(2 d)$, can be shown to be equal to $C$ plus a power series in $A^{2}$, where $\bar{V}$ is precisely the first-order correction in this series. $\bar{V}$ thus accounts for the amplitude-dependence of $V_{0}$.

\section{Conclusion}

We have presented a novel multiple-scales approach that incorporates the preservation of rotational symmetry embodied in NLH equations. It has been demonstrated, for the first time, that small-amplitude Helmholtz-Kerr dark solitons satisfy a KdV equation. Particular novelty arises from noting that the integrable $\mathrm{KdV}$ equation has been derived from the non-integrable 
NLH equation. A physical interpretation of this result can be uncovered by observing that the Helmholtz slow variables describe a soliton in a near-paraxial framework, and that in the paraxial limit this soliton does indeed satisfy an integrable wave equation.

Perturbations to the solitons may be analysed using KdV theory. For example, the potential use of quasi-linear solitons as information signals that are guided in re-configurable refractive waveguides (written to the medium by relatively intense optical beams) has greater promise when signals can be guided at arbitrary propagation angles.

The NLH equation considered is a generalization of the NLS equation through a generic modification to the linear wave operator. A connection has thus been established between the $\mathrm{KdV}$ equation and a wider class of nonlinear wave equations. Numerous other NLH equations can be shown to possess exact analytical soliton solutions [25]. Further new connections, involving such nonlinear wave equations, may be established and these may provide alternative analytical frameworks and novel insights in these cases.

Finally, we comment on a potential alternative approach to establishing a connection between Helmholtz-Kerr and KdV solitons. There exists a formalism for mapping solutions of a generalized KdV equation onto solutions of the NLS equation [29-31]. This is based on the fluid equations associated with the NLS system, derived by introducing a Madelung transformation. However, we have not found an equivalent mapping when the NLS equation is replaced by the NLH equation. A generalized procedure, such as that used by Fedele et al [29-31], is also unlikely to exist since the familiar fluid equations are not recovered in the Helmholtz case.

\section{References}

[1] Stegeman G I and Segev M 1999 Science 2861518

[2] Kivshar Y S 1998 Opt. Quantum Electron. 30571

[3] Zakharov V E and Shabat A B 1972 Sov. Phys._JETP 3462

[4] Zakharov V E and Shabat A B 1973 Sov. Phys._JETP 37823

[5] Kivshar Y S and Luther-Davies B 1998 Phys. Rep. 29881

[6] Kivshar Y S and Malomed B 1989 Rev. Mod. Phys. 61763

[7] Kivshar Y S 1990 Phys. Rev. A 421757

[8] Kivshar Y S 1990 Opt. Lett. 151273

[9] Kivshar Y S 1991 Opt. Lett. 16285

[10] Kivshar Y S 1991 Phys. Rev. A 431677

[11] Kivshar Y S 1991 Opt. Lett. 16892

[12] Remoissenet M 1999 Waves Called Solitons: Concepts and Experiments 3rd edn (Berlin: Springer)

[13] Kivshar Y S 1992 Opt. Lett. 171322

[14] Dodd R K, Eilbeck J C, Gibbon J D and Morris H C 1982 Solitons and Nonlinear Wave Equations (London: Academic)

[15] Infeld E and Rowlands G 2000 Nonlinear Waves, Solitons and Chaos 2nd edn (Cambridge: Cambridge University Press)

[16] Coullet P, Gil L and Rocca F 1989 Opt. Commun. 73403

[17] Coullet P, Gil L and Lega J 1989 Physica D 3791

[18] Mandel P 1997 Theoretical Problems in Cavity Nonlinear Optics ed P L Knight and A Miller (Cambridge: Cambridge University Press)

[19] Chi S and Guo Q 1995 Opt. Lett. 201598

[20] Crosignani B, Yariv A and Mookherjea S 2004 Opt. Lett. 111254

[21] Ciattoni A, Crosignani B, Mookherjea S and Yariv A 2005 Opt. Lett. 30516

[22] Chamorro-Posada P, McDonald G S and New G H C 2002 J. Opt. Soc. Am. B 191216

[23] Chamorro-Posada P, McDonald G S and New G H C 1998 J. Mod. Opt. 451111

[24] Chamorro-Posada P and McDonald G S 2003 Opt. Lett. 28825

[25] Christian J M, McDonald G S and Chamorro-Posada P 2006 Helmholtz-Manakov solitons, Phys. Rev. E at press Christian J M 2006 On the theory of Helmholtz solitons PhD Thesis University of Salford 
[26] Chamorro-Posada P and McDonald G S 2006 Spatial Kerr soliton collisions at arbitrary angles Phys. Rev. E 74 036609

[27] Satsuma J and Yajima N 1974 Prog. Theor. Phys. 55284

[28] Chamorro-Posada P, McDonald G S and New G H C 2000 J. Mod. Opt. 471877

[29] Fedele R, Schamel H and Shukla P K 2002 Phys. Scr. T98 18

[30] Fedele R and Schamel H 2002 Eur. Phys. J. B 27313

[31] Fedele R, Karpman V I and Shukla P K 2003 J. Phys. A: Math. Gen. 361169 